\title{
Archéopages
}

Archéopages Archéologie et société

41 | 10/2014-01/2015

La place du cheval

\section{Un cheval, des chevaux}

Propos recueillis par Catherine Chauveau

\section{Armelle Gardeisen, Roland Étienne et Catherine Chauveau}

\section{OpenEdition}

Journals

Édition électronique

URL : https://journals.openedition.org/archeopages/1048

DOI : $10.4000 /$ archeopages. 1048

ISSN : 2269-9872

Éditeur

INRAP - Institut national de recherches archéologiques préventives

\section{Édition imprimée}

Date de publication : 1 août 2015

Pagination : 74-79

ISSN : 1622-8545

Référence électronique

Armelle Gardeisen, Roland Étienne et Catherine Chauveau, «Un cheval, des chevaux », Archéopages [En ligne], 41 | 10/2014-01/2015, mis en ligne le 01 janvier 2017, consulté le 22 janvier 2022. URL http://journals.openedition.org/archeopages/1048 ; DOI : https://doi.org/10.4000/archeopages. 1048 


\section{Un cheval, des chevaux}

\section{Pégase, Bucéphale, le sacrifice de chevaux par Achille sur la tombe de Patrocle sont les exemples les plus fameux du prestige auquel est associé le cheval dans le monde méditerranéen antique. Mais cet animal a-t-il vraiment toujours un statut à part?}

\section{Armelle Gardeisen}

est archéozoologue au CNRS dans l'UMR 5140 «Archéologie des sociétés méditerranéennes». Parmi ses dernières publications : avec N. Boulbes, Diversité du cheptel équin en Gaule méditerranéenne à la fin du III s. av. J.-C. d'après l'étude des métacarpes d'équidés du site de PechMaho, actes du coll. Arles avril 2012, Monographie d'archéologie méditerranéenne hors-série n ${ }^{\circ} 6, \mathrm{p} 199$ 220, 2014 ; avec E. Furet et N. Boulbes (dir.), Histoire d'équidés: des textes, des images et des os, actes du coll. Montpelliermars 2008, Monographie d'archéologie méditerranéenne, hors-série $n^{\circ} 4,2010$; Les équidés dans le monde méditerranéen antique, actes du coll. Athènes,

Monographie d'archéologie

méditerranéenne hors-série $\mathrm{n}^{\circ} \mathrm{1}, 2005$.

\section{Roland Étienne}

est historien, spécialiste du monde hellénistique, ancien directeur de l'École française d'Athènes et professeur émérite à l'université de Paris 1. Parmi ses dernières publications : avec C. Müller et F. Prost (dir.), Archéologie historique de la Grèce antique, coll. L'Antiquité, une histoire, Ellipses, $3^{\mathrm{e}}$ éd mise à jour, 2014 ; La méditerranée au VII ${ }^{e}$ s. av. J.-C. : essais d'analyse archéologique, Travaux de la Maison René Ginouvès, De Boccard, 2010. 


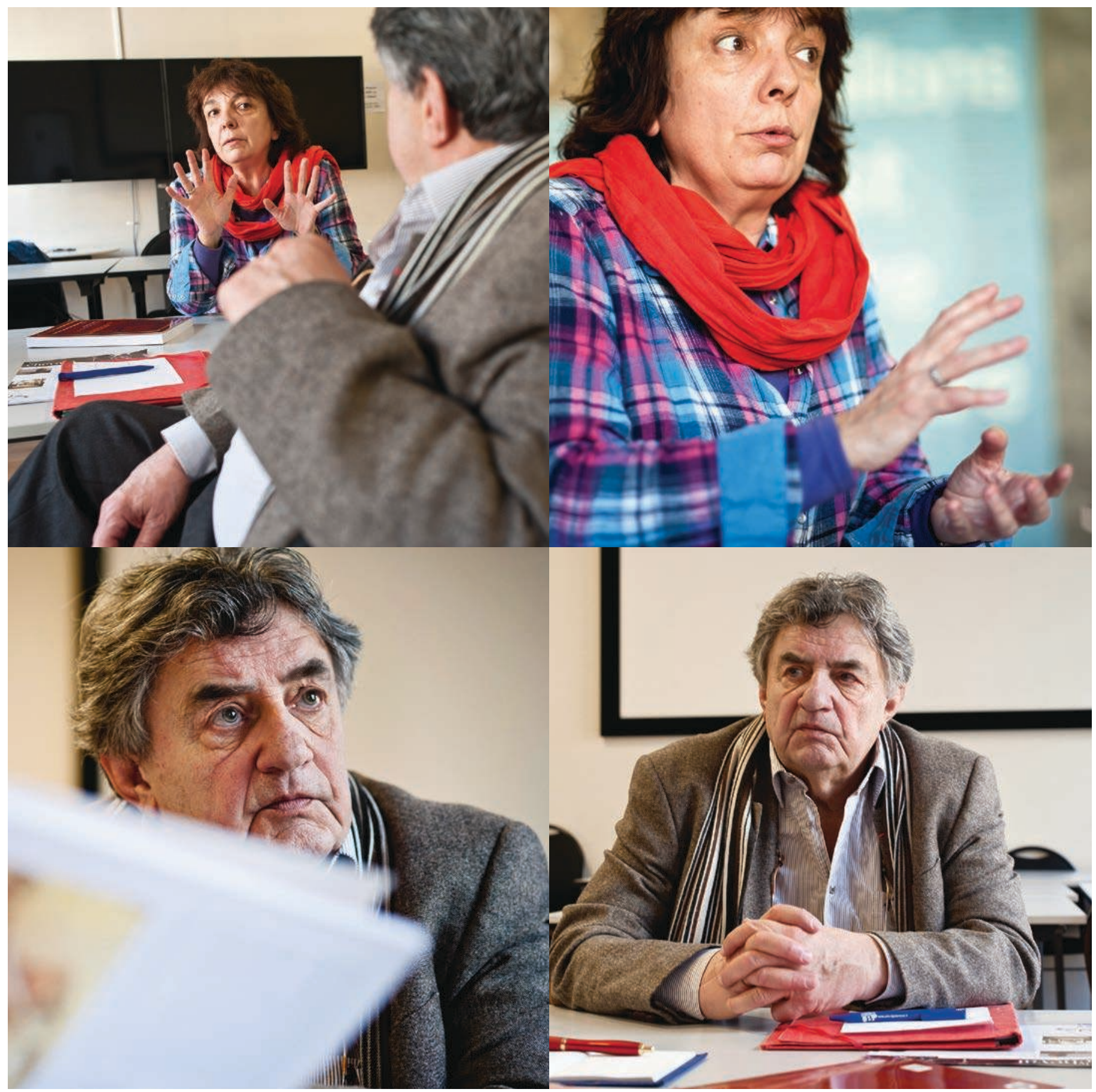


Roland Étienne Le cheval plus que tout autre est un animal particulièrement informé pour l'Antiquité : par des restes matériels, par des images, par des écrits. Son étude donne une extraordinaire occasion de rencontre entre divers spécialistes. De surcroît, on est face à un animal qui a un statut à part, dans la société, dans les mythes, dans les représentations... Le cheval appartient, à ma connaissance, aux premières représentations figurées sur céramique et ce dès l'époque minoenne. Et à partir du $\mathrm{IX}^{\mathrm{e}}$-VIII ${ }^{\mathrm{e}}$ siècle avant notre ère, en Grèce, les petites figurines de cheval comptent parmi les offrandes les plus répandues. On en a retrouvé un très grand nombre à Olympie, notamment, peut-être en lien avec l'installation des concours olympiques. Ce ne sont que quelques exemples, car le cheval est partout dans le monde grec antique!

Armelle Gardeisen Les visions de l'archéologue et de l'archéozoologue sont complètement à l'opposé. Pour nous, le cheval est l'animal invisible! Sauf dans les cas exceptionnels où on le retrouve dans des assemblages de type nécropoles, dépôts rituels etc. Comme le cheval ne passe pas réellement dans le circuit alimentaire, on en a une image extrêmement floue. S'ajoute à cela une autre difficulté d'ordre archéozoologique : dans cette grande famille de mammifères, la très complexe famille des équidés pose un problème. Elle est en effet constituée d'animaux très proches morphologiquement. La différence entre un âne et un cheval se voit, c'est vrai, mais entre un petit cheval et un grand âne, déjà moins. D'un point de vue strictement ostéologique, et au niveau dentaire, il y a des critères distinctifs qui permettent de trancher entre asiniens et cabalins. Mais sur des ossements, mal conservés, éparpillés, fragmentés etc. il faut vraiment avoir de la chance pour avoir un élément porteur d'un de ces critères! Pour les périodes antiques, on ne se pose pas plus de questions d'ordre spécifique mais ces difficultés vont empirer pour les périodes où se développent les pratiques d'hybridation. L'homme en plein processus domesticatoire modifie avec une idée, un objectif. Et tous les croisements ont pu lui passer en tête en fonction des équidés qu'il avait sous la main. On peut avoir une certaine image de la morphologie globale des chevaux grâce à l'iconographie dans le monde méditerranéen et bien au-delà, en Asie, etc. Mais reste le souci qu'on ne peut passer d'une image à un os ! Quand on a des individus entiers, on travaille avec des méthodes de morphométrie, d’ostéologie comparée sur les probables masses musculaires, déformations, castrations... Et on constate et on mesure des variations dans l'allure générale du squelette, qui ne peuvent plus, comme au bon vieux temps du Paléolithique, être imputées à des changements environnementaux. À partir d'os ou de dents, on estime ce que pouvait être le squelette couvert de muscles et de poils. Parmi les critères de mesure, qui viennent en grande partie de la paléozoologie, le plus utilisé est la hauteur au garrot, établi sur des longueurs d'ossements, donc sur des os longs entiers, ce qui est une denrée rare pour les archéozoologues ! On se base donc sur des os plus souvent intacts, métacarpiens et métatarsiens, et grâce à une petite équation très simple réalisée à partir d'études de populations de chevaux contemporains, on calcule une estimation de hauteur au garrot. La mesure de la hauteur au garrot permet à tous de se représenter la taille du cheval et c'est ce qui la rend plus appréciable. Mais ce n'est qu'une estimation basée sur un seul os, et on sait très bien, quand on fait un peu d'évolution et d'ostéologie, que cela ne sera qu'une valeur relative. Le squelette est un système complexe : deux individus peuvent avoir la même taille alors que leurs os longs ont des tailles différentes. Il y a des relations intra-squelettiques qui font que la morphologie change. Les variations intrasquelettiques sont souvent liées à une adaptation fonctionnelle; pour le cheval, c'est le plus souvent la locomotion, en lien avec le type de terrain sur lequel il court, et par conséquent la vitesse. Un animal très adapté à la course a de très longs métapodes et les premiers segments des membres plutôt courts. Mais s'il court dans la steppe ou s'il court dans la tourbe, il aura des proportions squelettiques complètements différentes. Il faut donc absolument croiser tous les types d'informations disponibles et ne pas tout fonder sur une seule. À conditions d'élevage égales, les animaux évoluent plus ou moins de la même manière en fonction de leurs caractères intrinsèques. Et dans des conditions d'élevage différentes, il peut y avoir des variations qui tiennent à ce que l'un mange d'une telle manière, l'autre est dehors toute la journée, l'autre ne sort pas de l'écurie, l'un aura à boire à volonté, l'autre boira de l'eau stagnante... Ils seront plus ou moins protégés des épizooties, des parasites, etc.

RE Que de précautions à prendre, en effet, sur l'interprétation de la taille des chevaux, et notamment sur les rapports hâtifs entre élevage, race et représentations figurées et que d'intérêt à croiser divers types de données ! Dans un article très intéressant, Henri-Paul Francfort et Sébastien Lepetz ${ }^{1}$ étudient les chevaux représentés dans les processions de l'Apadana de Persépolis, qui ont des caractères et des tailles très différents. On avait beaucoup glosé, et tiré des conclusions rapides, pour essayer de retrouver des races, des types d'élevages et d'identifier le fameux cheval niséen, nec plus ultra des élevages perses ! Mais les archéologues font la preuve que les variations de tailles sont en rapport avec une idéologie et non pas avec des races ou des provenances : les grands chevaux sont liés aux grands personnages. Il est possible qu'il y ait aussi des valeurs idéologiques dans les robes représentées. Bucéphale monté par Alexandre sur la mosaïque de Pompéï est un alezan ${ }^{2}$ et tous les chevaux des «vilains » Perses sont noirs. Mais l'homme instaure bien une hiérarchie au sein 


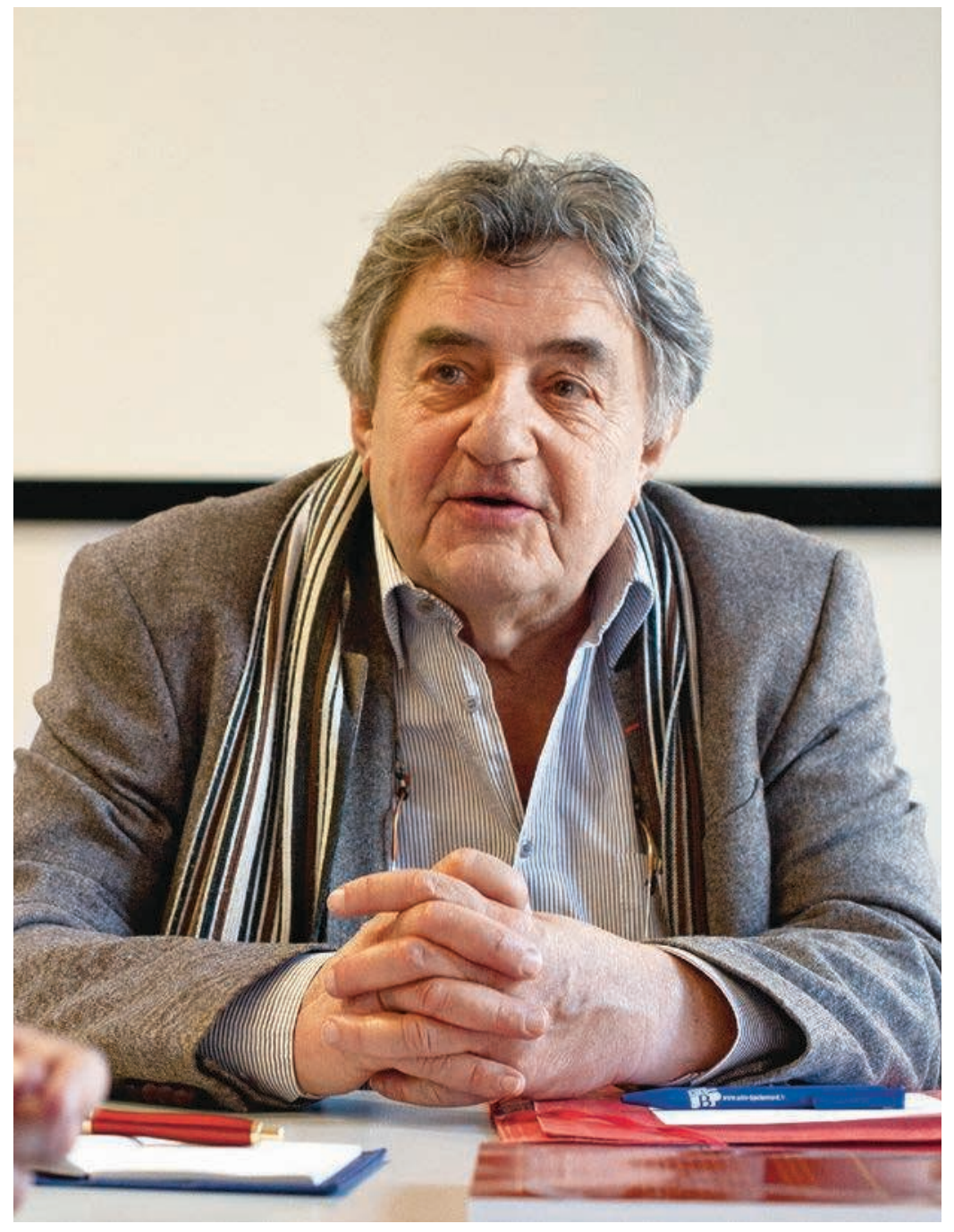

\section{Le cheval, support de prestige, est l'objet d'un attrait, tout comme le cavalier.}

\author{
Roland Étienne
}

des équidés. Lâne est en bas, et pourtant c'est le plus résistant. Les mules sont assez prisées ; il y avait des concours de mules à Olympie. Cela nous amène à la question du début de la domestication des équidés et de l'hybridation. Où en est-on et quelles sont les plus anciennes attestations d'équidés en contexte anthropique?

AG La question du moment et lieu de la domestication ne fait pas l'unanimité. Des sites de steppes nord asiatiques, datés $d u I^{\mathrm{e}}$ millénaire, ont livré des restes de chevaux. Et l'un d'eux, Dereikva, en Russie, a été considéré comme l'un des premiers foyers de domestication de chevaux parce que l'on y avait trouvé un objet sculpté dans un andouiller de cervidé, qui a été interprété comme un mors. Mais on ne peut démontrer ostéologiquement que les ossements sont ceux d'animaux domestiqués. Non seulement cet objet aurait pu être une pièce d'un autre outillage mais en plus il est un peu rapide d'établir que les débuts de la domestication du cheval ont aussi été ceux de l'invention du mors. Le cheval est un animal lourd, puissant, peureux. L'approcher n'est pas évident ; le capturer avec un cordage n'est possible qu'avec de l'entraînement, et puis après, il faut le tenir ! Et là, comme dit Jean-Pierre Digard ${ }^{3}$ avec humour : le cheval galope et le type vole! Cependant, grâce aux travaux de Véra Eisenmann ${ }^{\mathbf{4}}$, entre autres, la discussion porte plus sur la détermination ostéologique d'un cheval domestique versus celle d'un cheval sauvage. Ce n'est pas si simple. Il est très important de combiner des informations d'ordre différent. Et puis il faut se méfier de notre tendance à croire que les évolutions techniques sont systématisées et utilisées majoritairement. Il peut y avoir toutes sortes de décalages : on sait qu'au XIX ${ }^{\mathrm{e}}$ siècle, moment où les harnachements sont parfaitement sophistiqués, les chevaux miniers pouvaient être attachés n'importe comment aux wagons. Pour revenir au monde grec et quitte à élargir le sujet aux ânes, les premières présences de restes d'équidés sont les sites de Tsoungiza, dans le Péloponnèse, et de Cnossos, où on trouve des traces de consommation dès l'âge du Bronze ancien. Dans les assemblages fauniques de sites d'habitat (assemblages de restes de consommation), il y a de temps en temps un os de cheval. Est-ce que ce sont des éléments de type intrusif arrivés de façon mécanique dans un mouvement de sédiments ? Pourquoi pas, mais c'est assez récurrent pour que l'on s'interroge. À partir de la période archaïque, on ne les voit plus dans le régime alimentaire. D'un point de vue archéozoologique, il y a donc un bruit de fond « cheval» mais pas forcément des attestations comme on les aime, avec des profils anatomiques, des courbes de mortalité, des traces bien localisées, bien répétitives etc. Et dans le monde occidental, c'est un peu la même chose. Dès que l'on s'aperçoit que, à coup sûr vers le $\mathrm{VI}^{\mathrm{e}}$ siècle avant notre ère, le cheval n'est pas élevé pour sa viande, on peut évoquer ces essais de création d'individus que sont 
les mules, qui ont les avantages de l'âne et ceux du cheval sans en avoir les inconvénients. Mais les gènes qui signent les variations de couleurs des robes ont été repérés autour de 3000 ans avant notre ère. Cela incite à penser qu'il y a une connaissance suffisante de l'animal bien en amont des pratiques d'hybridation parce que les hommes ont commencé à faire des essais de croisement, par exemple pour obtenir des types de comportements, de puissance, d'allure... aussi bien sur le cheval que sur l'âne. On peut penser que progressivement ils atteignent un niveau où ils peuvent travailler au sens zootechnique du terme sur l'espèce, sur l'animal. Et que c'est peut-être à ce moment là qu'ils « fabriquent » des chevaux de prestige. RE Comme ceux que l'on trouve sacrifiés dans les tombes dites royales d'une période allant de 1500 avant notre ère jusqu'à l'époque classique, à Chypre, chez les Macédoniens... On n'a pas toujours de restes osseux mais parfois des éléments du harnachement.

AG Les chevaux sont présents de deux façons : ou bien enterrés entre des sépultures humaines au sein d'une nécropole, ce qui fait que l'association homme/cheval n'est pas évidente ; ou bien dans les couloirs d'accès aux dromos, par couple, attelés ou non. Et là, le cheval est un des signes de la richesse du défunt, et il est lui-même porteur d'éléments de prestige.

RE On a de magnifiques tapis de selle, par exemple. Le cheval représenté sur les céramiques mycéniennes est un cheval apprêté, coiffé et vêtu. Il participe à une mise en scène, à côté des hommes. Il a manifestement une place à part. Chez les Thraces, les chevaux royaux portaient des caparaçons très richement décorés de représentations tout à fait étonnantes, mythologiques et fantastiques ; on a notamment la représentation d'une hiérogamie très réaliste. À mon avis, ce sont des éléments de parade. La richesse, c'est d'avoir un cheval et les moyens de le décorer avec des matières nobles et chères. Tout cela montre l'intérêt et le soin portés au cheval de l'époque archaïque à l'époque romaine, en Grèce. Plus généralement, il y a trois emplois du cheval : le transport, le concours et la guerre, qui est la question essentielle partout jusqu'au XIX ${ }^{\mathrm{e}}$ siècle. Dans la Grèce antique, les cavaleries militaires sont très importantes. On a des traités d'hippiatres datés du IV siècle, notamment celui d'Apsyrtos. Les hippiatres devaient non seulement soigner les chevaux mais aussi aider à les sélectionner. Les chevaux les plus recherchés alors étaient ceux des races espagnoles et perses, mais, à notre surprise, Apsyrtos dénigre les chevaux thraces, si souvent représentés de façon valorisante et très prisés à d'autres époques. Est-ce que c'était pour détourner les achats vers d'autres régions où il avait des intérêts? On sait par les textes qu'existent de grandes régions d'élevage, comme la Thessalie. Le thessalien Ménon de Pharsale, par exemple, vient aider les Athéniens au $\mathrm{V}^{\mathrm{e}}$ siècle en amenant 300 cavaliers pris parmi ses dépendants. C'est énorme d'entretenir 300 cavaliers! Il faut à peu près un hectare par an pour nourrir un cheval. Autre exemple connu, la cité d'Argos qui avait ses propres haras et vendait ses chevaux. On le sait par une anecdote : Alcibiade ${ }^{\mathbf{5}}$ a joué un tour pendable à un de ses amis ; il lui a conseillé d'acheter un cheval à Argos et l'a fait courir sous son propre nom. L'élevage et le contrôle de ces animaux à Athènes est aussi connu grâce à l'étude de centaines de lamelles de plomb archivant les inspections de la cavalerie de guerre. Elles donnent le nom du cheval, celui de son propriétaire, la couleur de sa robe et sa valeur. Les valeurs de ces chevaux sont comprises entre 100 et 1200 drachmes, important écart dû au fait que le cheval se déprécie chaque année : on commençait à 1200 drachmes; on enlevait vraisemblablement 100 drachmes chaque année et, au bout de 10 ans, le cheval était réformé. Pour se faire une idée de cette valeur, comparons-la à la solde d'un soldat, soit une drachme par jour. Cela en fait des mois de salaire ! Certains chevaux extraordinaires auraient atteint des prix exorbitants. Il est dit que Bucéphale valait de 13 à 16 talents! Un talent équivaut à 6 ooo drachmes, une drachme à 3,40 gr d'argent, cela fait donc à un peu près 26 kilos d'argent. Mais il est possible que ces chiffres soient exagérés pour forger la légende. Mais tout ceci n'est qu'une partie de ce que l'on peut comprendre de l'élevage. Où sont les enclos, les écuries et autres équipements équestres?

AG Les vestiges archéologiques concernant l'élevage sont tout aussi rares que les restes osseux. Mais que chercher? Il faut sortir du schéma moderne, « violet-leducien », de grandes écuries construites et rationalisées. De la préhistoire récente jusqu'à l'Antiquité, on n'a rien de ce genre alors que les opérations archéologiques se sont multipliées. Qu'en déduire ? Que les chevaux sont dehors, près des habitats, et au mieux protégés par des aménagements périssables en bois ou en roseau. On n'a donc pas de signal réel de l'élevage. En archéozoologie, il y a un critère qu'on utilise beaucoup qui est le taux de présence de juvéniles. Dans les sites archéologiques, on a des petits moutons, des petits cochons, des petits chiots etc. et jamais de petits poulains! Lorsque l'on trouve du cheval, ce sont des adultes (dans presque $98 \%$ des cas!). Ces animaux sont-ils donc « introduits » à l'état adulte? Mais il y a une exception : dans la forteresse du premier âge du Fer de Vilars, en Catalogne, des fœetus de chevaux ont été inhumés dans une douzaine de maisons, en association plus ou moins régulière avec des périnatals humains ${ }^{\mathbf{6}}$ (inhumer les enfants mort-nés dans l'habitat étant une grande tradition de l'âge du Fer). Autant de cas avérés, cela veut dire que les naissances, ou en tout cas les juments, sont surveillées et contrôlées. Car si on considère que les troupeaux de chevaux sont hors les murs, autour de la ville, un poulain mort-né est la proie de divers prédateurs et disparaît dans l'heure qui suit. Dans cette région, 


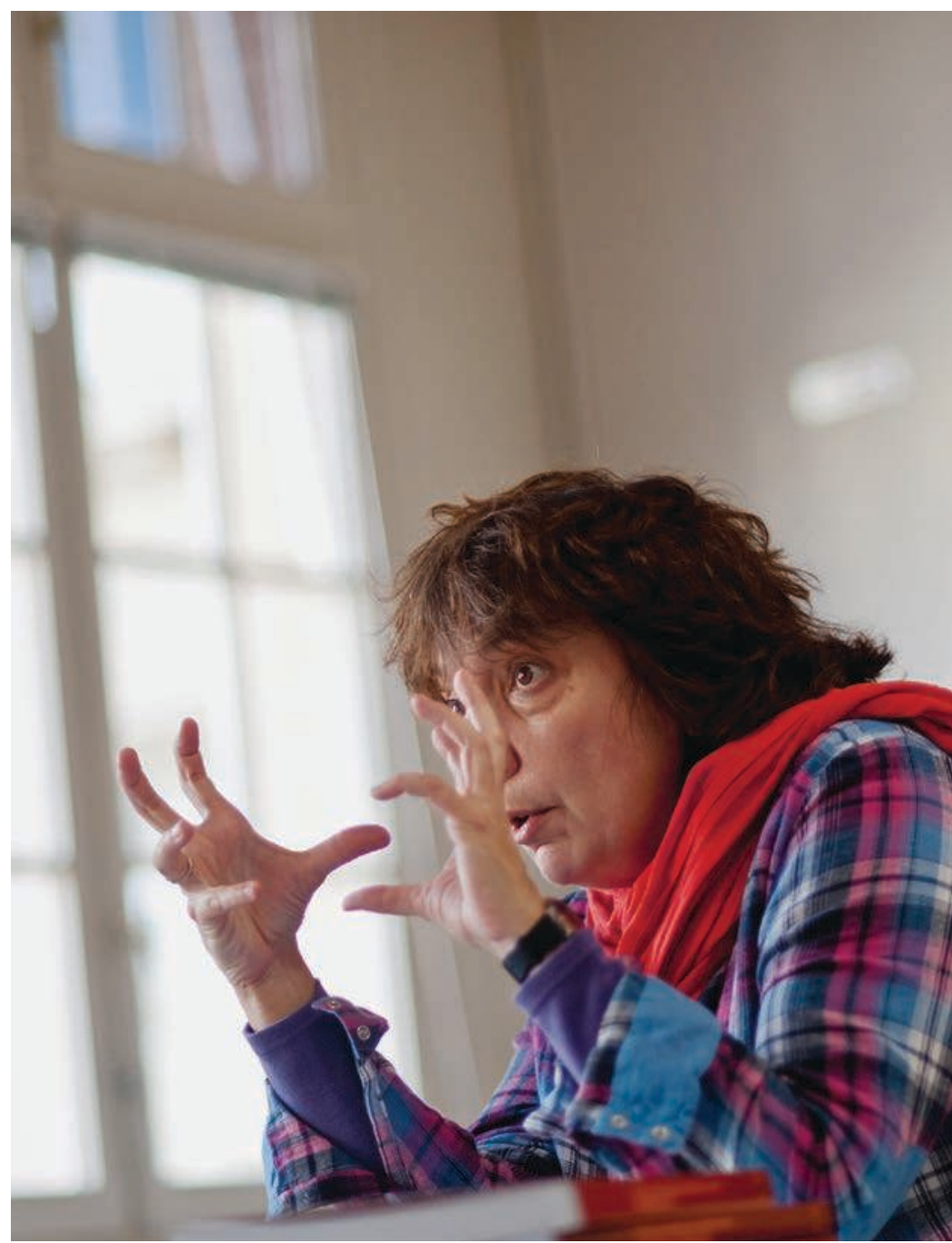

\section{Bien distinguer les équidés à affinité asinienne de ceux à affinité cabaline est déjà un problème.}

Armelle Gardeisen connue par les textes pour ses élevages de chevaux, on a observé cette pratique d'inhumation sur deux autres sites. On ne peut à proprement parler ici de sélection des populations de chevaux mais en tout cas les juments gravides sont sous contrôle d'une manière ou d'une autre. Est-ce que c'est cela l'élevage? Je pense que oui! Les troupeaux restent dehors mais on s'en occupe, on les soigne, on va les voir tous les jours, etc. Dans le même site, les déchets de consommation sont constitués à $3 \%$ de restes de chevaux ; ce fameux signal ténu, cet arrière-fond, observé presque partout. Donc si l'on n'avait pas eu ces fotus, à aucun moment on se serait posé la question de l'élevage des chevaux à l'époque archaïque, au début de l'âge du Fer. Sur le site de Pech Maho ${ }^{7}$, on a une population de chevaux qui sont morts quasiment au même moment, tous âgés entre 5 et 12 ans, âges de travail pour un cheval. Car dans la valeur de l'animal, il y a tout le temps qu'on passe à l'entretenir et l'éduquer avant de pouvoir s'en servir! Ces chevaux sont morts au combat, autour de 200, on pense en relation avec la deuxième guerre punique.

RE On attend l'étude d'épaves ayant servi au transport de chevaux, dans le cadre de cet élevage pour le commerce ou de la formation de cavaleries de guerre. Le transport d'équidés par voie maritime ou terrestre devait être organisé, d'une façon ou d'une autre. Comment voyageaient les chevaux que l'Égypte achetait dans le Péloponnèse? Et les champions que l'on faisait courir à Olympie tous les quatre ans? Et les cavaleries de guerre au gré des conflits et des alliances? Thucydide, notamment, fait des allusions très précises, au $\mathrm{V}^{\mathrm{e}}$ siècle, au transport de ces chevaux par bateaux. Il évoque les box et les difficultés concrètes. Ce n'est pas rien d'aménager les navires pour pouvoir caser, nourrir, surveiller tant de chevaux, évacuer leurs excréments, éviter la pagaille et les accidents. Cela pose de réels problèmes, surtout quand on connaît la valeur économique de ces animaux. AG N'oublions pas qu'il peut y avoir au sein d'une même société des chevaux qui ont des fonctions et donc des statuts différents. Â une époque où le cheval est l'image d'une élite guerrière dans le monde méditerranéen et occidental et le reflet d'une puissance nouvellement acquise (ce qui correspond à la multiplication de ses représentations), il y a toujours des gens qui vivent normalement et d'autres chevaux qui triment et passent dans le circuit alimentaire. C'est ce que l'on constate à l'époque romaine, en Gaule, où l'on observe que des chevaux sont débités et certains os récupérés pour élaborer des objets, car les métatarsiens de chevaux constituent une appréciable matière première. Se côtoient donc des chevaux choyés à qui on donne des honneurs funéraires et d'autres qu'on exploite, dans tous les sens du terme. 